\title{
Does it need to perform anterior column support after Smith-Petersen osteotomy for ankylosing spondylitis?
}

\author{
Ki-Tack Kim • Dae-Jean Jo $\cdot$ Sang-Hun Lee $\cdot$ \\ Kyoung-Jun Park $\cdot$ Jae-Heung Sin
}

Received: 6 September 2010/Revised: 1 July 2011/Accepted: 31 August 2011 / Published online: 20 September 2011

(C) The Author(s) 2011. This article is published with open access at Springerlink.com

\begin{abstract}
Purpose The aim of this study was to determine whether anterior column support is required in Smith-Petersen osteotomy procedure with correction angles of more than $10^{\circ}$, while examining the subsequent healing patterns in relation to the disrupted area.

Methods An analysis was done on 26 segments of 19 patients who showed a correction angle of more than $10^{\circ}$ in the anterior opening after SPO. There were 17 male and two female patients with a mean age of 40 years (24-56 years). The mean follow-up period was 6.5 years (2-9.1 years). The patients were classified according to the site of the anterior opening, as the disc level, the lower end-plate of the upper body (upper body), or the upper end-plate of the lower body (lower body). The healing patterns of anterior opening and the radiological correction angles were evaluated relative to the opening site.

Results In all cases, bony fusion was confirmed at a mean period of 5.6 months (3-6.7 months) after surgery and the anterior opening gap was healed in 18 segments (69.2\%). For patients that developed an opening in the upper body,
\end{abstract}

K.-T. Kim · S.-H. Lee $\cdot$ J.-H. Sin

Department of Orthopedic Surgery, Spine Center, Kyung Hee University East West Neomedical Center, Seoul, Korea

D.-J. Jo ( $\square)$

Department of Neurosurgery, Spine Center, Kyung Hee University East West Neomedical Center, \#149, Sangil-dong, Kangdong-gu, Seoul 134-090, Korea e-mail: apuzzo@daum.net

K.-J. Park

Department of Orthopedic Surgery, National Medical Center, Seoul, Korea all of the gaps were healed. The gaps in the lower body opening group were healed in $85.7 \%$ of the cases, and for the opening at the disc level, the gaps were healed only in $12.5 \%$ of the cases. The least amount of correction was obtained when anterior opening occurred in disc level.

Conclusions In our study of subjects presenting with anterior opening angles from $10^{\circ}$ to $32^{\circ}$, we obtained successful fusion without the need for additional anterior interbody fusion. Improved gap healing and increased correction angles were obtained when the opening was present in the upper or lower body endplates compared to those at the disc space level.

Keywords Ankylosing spondylitis - Kyphotic deformity · Smith-Petersen osteotomy

\section{Introduction}

Smith-Petersen osteotomy utilizes the posterior margin of the body as the pivot, with the posterior column closed and the anterior column opened. With sufficient elasticity in the intervertebral disc, a certain degree of lengthening can be achieved during surgery. However, when all discs are fully ossified, as in AS patients, elasticity is absent. In such cases, anterior opening may occur when disruption of the disc happens or osteoclasis of the end plate develops [1]. When the angle of opening is $\sim 30^{\circ}$, there are substantial defects of the anterior column, thus calling into question the necessity for anterior gap reconstruction. Earlier studies rarely mention such themes while some authors have emphasized the need for combined anterior and posterior procedures [1-5].

With 15 years of experience, comprising more than 300 cases of patients with AS, we previously reported the need 
for reconstruction of the anterior column in cases of SPO on segments with pseudarthrosis [6]. However, questions arose regarding the usage of SPO on fully ossified segments. With small gaps, bone union is easily accomplished (as is characteristic of AS), with good healing results. However with gaps larger than a certain degree, the indications for the need for anterior reconstruction seemed questionable. We also felt the obliged to take into account the differing healing patterns which depend on the disruption sites.

The aim of this study is to determine whether anterior column support is required in SPO procedure with correction angles of more than $10^{\circ}$, while examining the subsequent healing patterns in relation to the disrupted area.

\section{Materials and methods}

Corrective osteotomy for kyphotic deformity was performed in 327 AS patients from April 1995 to August 2008. SPO was performed on 62 segments of 54 of these patients.

The indications for corrective surgery were as follows: patients with ankylosis of the cervical vertebrae that hindered forward gazing, patients complaining of cosmetic problems and lastly patients who were easily fatigued due to kyphotic deformity. SPO was performed at the level that required a correction angle of $10^{\circ}-20^{\circ}$. In the case of pseudarthrosis, SPO was performed on the lesion segment, and anterior reconstruction was also carried out (19 segments in 12 patients).

Of 54 patients who underwent SPO, patients with a correction of less than $10^{\circ}$ and those who received anterior reconstruction because of pseudarthrosis were excluded from this study.

An analysis was done on 26 segments of 19 patients who showed a correction angle of more than $10^{\circ}$ in the anterior opening of the surgery segment. Eleven of these 19 patients received SPO alone, while eight patients received SPO in combination with pedicle subtraction osteotomy in the lumbar region, due to insufficient correction after PSO. Usually, surgical plan was decided preoperatively and we performed PSO prior to SPO in most of cases. Study subjects included 17 male and two female patients with a mean age of 40 years (24-56 years) (Table 1$)$. The mean follow-up period was 6.5 years (2-9.1 years).

Visual analog scale (VAS) score and modified SRS 24 instrument were clinically assessed prior to surgery and at the last follow-up. Complications were also analyzed. In the radiological evaluation, the sagittal angle of the disc (DSA), the segmental angle (SA), and the C7 plumb line were analyzed prior to surgery, after surgery and at the
Table 1 Chosen osteotomies and osteotomy level

\begin{tabular}{llll}
\hline Patient no. & Sex/age & SPO level & PSO level \\
\hline 1 & $\mathrm{M} / 49$ & T12-L1 & - \\
2 & $\mathrm{M} / 29$ & $\mathrm{~L} 1-2$ & - \\
3 & $\mathrm{M} / 31$ & T11-12, L2-3 & - \\
4 & $\mathrm{M} / 26$ & T10-11, L1-2 & - \\
5 & $\mathrm{M} / 38$ & T11-12, L1-2 & - \\
6 & $\mathrm{M} / 46$ & T8-9 & $\mathrm{L} 2$ \\
7 & $\mathrm{M} / 37$ & T12-L1 & $\mathrm{L} 3$ \\
8 & $\mathrm{M} / 33$ & T12-L1 & $\mathrm{L} 3$ \\
9 & $\mathrm{M} / 46$ & T11-12, L1-2 & - \\
10 & $\mathrm{M} / 45$ & T11-12, L1-2 & L3 \\
11 & $\mathrm{M} / 56$ & T12-L1, L2-3 & - \\
12 & $\mathrm{M} / 53$ & T12-L1 & L3 \\
13 & $\mathrm{M} / 36$ & T12-L1 & L3 \\
14 & $\mathrm{~F} / 31$ & T12-L1 & L3 \\
15 & $\mathrm{M} / 37$ & T12-L1 & - \\
16 & $\mathrm{~F} / 26$ & T11-12 & L2 \\
17 & $\mathrm{M} / 43$ & T12-L1, L2-3 & - \\
18 & $\mathrm{M} / 48$ & T10-11 & - \\
19 & $\mathrm{M} / 24$ & L1-2 & - \\
\hline
\end{tabular}

SPO Smith-Petersen osteotomy; PSO pedicle subtraction osteotomy

final follow-up (Fig. 1). At the every follow-up visits, the fusion state was analyzed in order to evaluate the bony fusion. The criteria of bony fusion were determined as with a bony bridge in the anterior gap, $<5^{\circ}$ of motion in flexion and extension in the simple lateral view without radiolucent lesion around the screw.

The patients were classified according to the site of the anterior opening, as the disc level, the lower end-plate of the upper body (upper body), or the upper end-plate of the lower body (lower body) (Fig. 2). The healing patterns of anterior opening were classified as no change and ossification, and the radiological correction angles and loss of correction angles were evaluated relative to the opening site. Radiological analysis was surveyed by three separate spine specialists in the department of spinal orthopedics and neurosurgery.

An ANOVA test (variance test) was performed to determine whether the correction angle and healing differed according to the opening level. Any differences among the groups were assessed using Duncan's post-hoc test.

The distances from the apex to the disc level, the lower end-plate of the upper body (upper body), and the upper end-plate of the lower body (lower body) were measured and the differences in their correction angles and healing were analyzed, using ANOVA with Duncan's post-hoc test. For statistical analysis, SAS version 9.1 was used, and a $p$ value of $<5 \%$ was considered to be significant. 
Fig. 1 Radiographic measurement methods. a Sagittal angle of disc (DSA) and $\mathbf{b}$ segmental angle
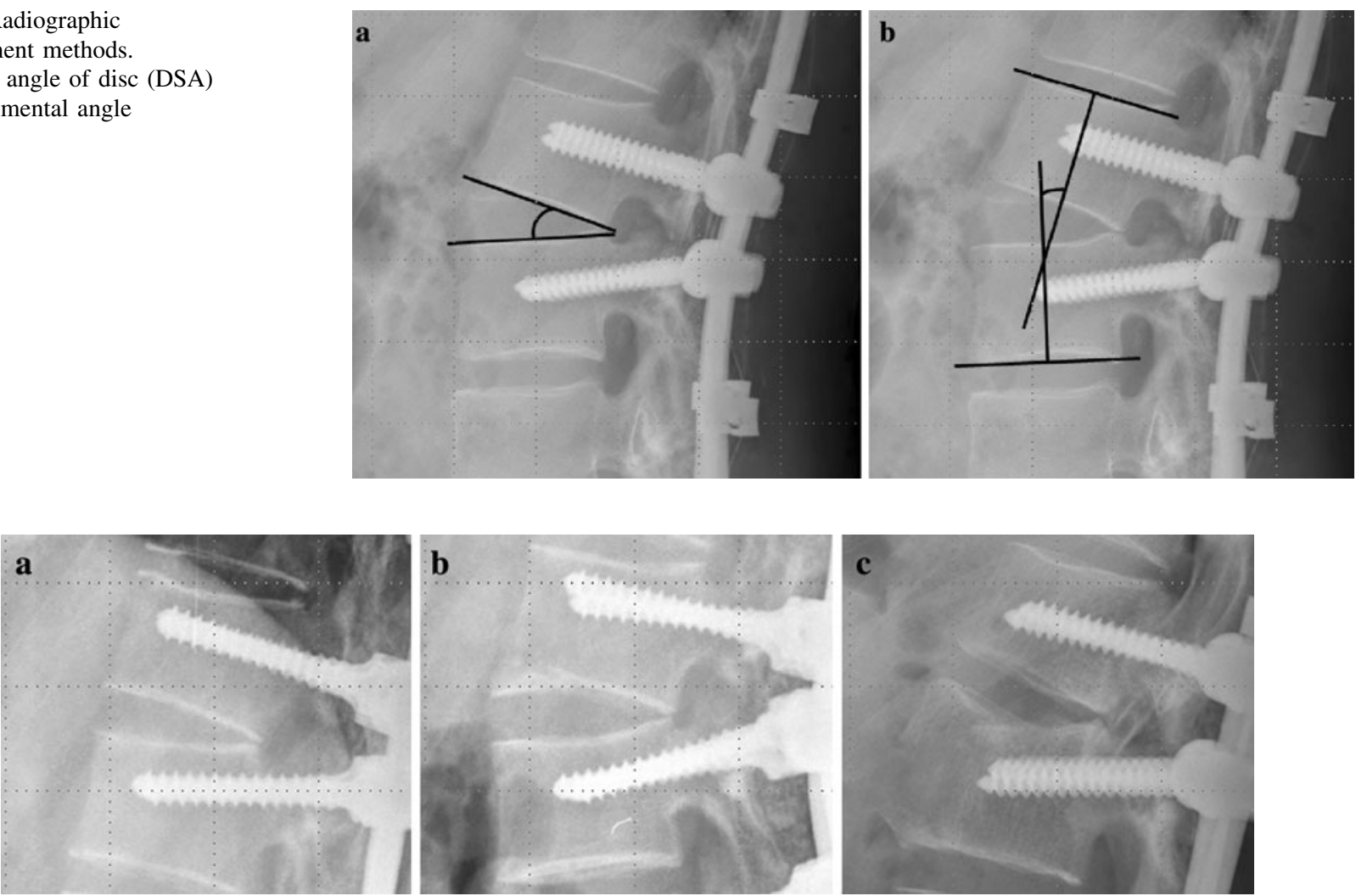

Fig. 2 Three anterior opening level. a Disc level, b lower endplate of upper body and $\mathbf{c}$ upper endplate of lower body

\section{Surgical technique}

To assess possible injuries to the spinal cord or spinal nerves during surgery, electrophysiological monitoring of somatosensory-evoked potentials and motor-evoked potentials was carried out under general anesthesia in all patients. A bronchoscope-assisted intubation was performed when an endotracheal intubation was impossible because of complete loss of neck motion. We used an operating table that allowed for adjustment of flexion and extension. To assure that the apex of kyphotic deformity was located centrally, the chest and iliac crests were fixed firmly in a prone position using a silicone bolster and sponge pads. Using a posterior approach, the segments to be corrected were exposed both superiorly and inferiorly, and the pedicle screws were then inserted. In most of cases, pedicle screws were inserted above 2-3 levels and below 2 levels at the osteotomy site. However, number of fixation points cephalad and caudad to the osteotomy site was different according to individual patient's situation. Structures such as spinous processes, lamina and facet joints were removed in an oblique manner from the area in which the osteotomy would be performed. With firm grasping of the cranial and caudal spinous processes with towel clamps, the operating table was extended gradually, at the same time closing the osteotomy site carefully. In cases in which satisfactory deformity correction could not be obtained by a single SPO, additional SPO was carried out (Table 1). Upon satisfactory deformity correction, posterior fusion using local bone obtained from the osteotomy site that involved lamina and fused facet joint were applied over the entire levels of the corrected segments after decortication with osteotome. If there was substantial defect, we covered this area with flat block bone. Anterior interbody fusion was not performed in all cases. After three to seven days of postoperative bed rest, patients were ambulated in TLSO braces. The braces were used for at least three months, at which time antero-posterior and lateral radiographs of the lumbar or thoracolumbar spine and standing lateral radiographs of the entire spine were obtained.

\section{Results}

Eleven patients underwent only SPO, and eight additional patients underwent SPO and subsequent PSO. SPO was performed on a total of 26 segments, with 12 patients having undergone SPO on one segment only, whereas seven patients underwent SPO on two segments each. The most common site of SPO was T12-L1, occurring in nine segments, followed by L1-2 for six segments, T11-12 for 

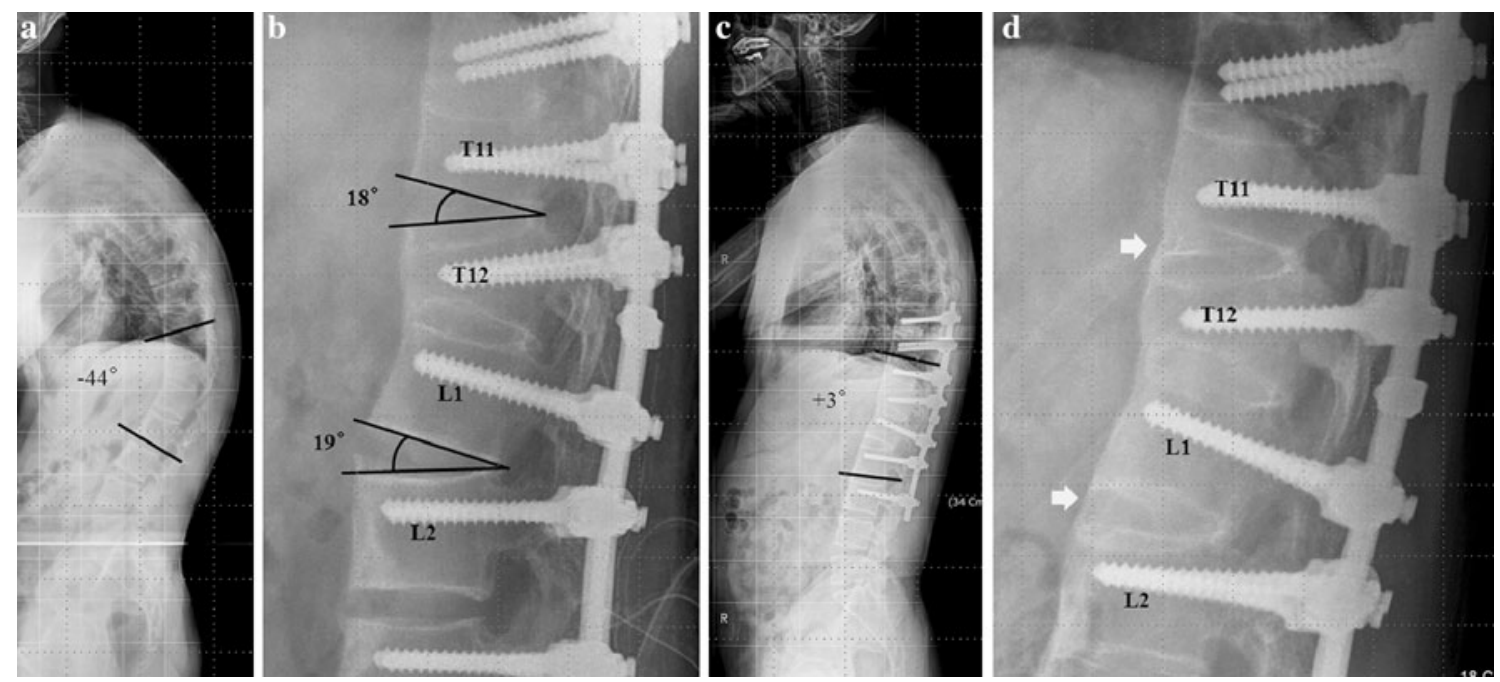

Fig. 3 A 46-year-old man with kyphotic deformity. a Local kyphosis caused by ankylosing spondylitis was $44^{\circ}$. b Lateral view after Smith-Petersen osteotomy at T11-12 and L1-2, sagittal angle of disc at T11-12 and L1-2 was $18^{\circ}$ and $19^{\circ}$, respectively. c Whole spine

five segments, L2-3 for three segments, T10-11 for two segments, and T8-9 for one segment (Table 1; Fig. 3).

Radiological evaluation revealed that the average sagittal angle of the disc prior to surgery was $-0.1^{\circ}\left(-7^{\circ}\right.$ to $\left.8^{\circ}\right)$ and was $20.1^{\circ}\left(10^{\circ}-31^{\circ}\right)$ after surgery. This angle was $18.7^{\circ}\left(11^{\circ}\right.$ to $\left.30^{\circ}\right)$ at the final follow-up. The average segmental angle prior to surgery was $-12.8^{\circ}\left(-34^{\circ}\right.$ to $\left.1^{\circ}\right)$. After surgery it was $8.8^{\circ}\left(-10^{\circ}\right.$ to $\left.22^{\circ}\right)$, and at the final follow-up, it was $7.6^{\circ}\left(-12^{\circ}\right.$ to $\left.21^{\circ}\right)$. The average $\mathrm{C} 7$ plumb line was $149.7 \mathrm{~mm}$ prior to surgery, $42.4 \mathrm{~mm}$ after surgery, and $45.2 \mathrm{~mm}$ at the final follow-up (Table 2).

The site of the anterior opening was divided into the disc level, the lower end-plate of the upper body (upper body), and the upper end-plate of the lower body (lower body). Of the 26 total segments, 11 segments had an opening in the upper body while 8 segments had an opening in the disc level and 7 segments in the lower body. In all cases, bony fusion was confirmed at a mean period of 5.6 months (3-6.7 months) after surgery and the anterior opening gap was healed in 18 segments $(69.2 \%)$. For patients that developed an opening in the upper body, all of the gaps were healed (100\% of the cases) after surgery. The gaps in lateral view at 3 years and 4 months after surgery showing improved sagittal imbalance and no loss of correction. d Lateral radiograph showing complete fusion at T11-12 and L1-2 (arrows) from the growth of bridge bone

the lower body opening group were healed in $85.7 \%$ of the cases, and for the opening at the disc level, the gaps were healed only in $12.5 \%$ of the cases (Table 3). Notably, according to the opening level of each group, these gap healings (anterior ossification) showed a statistically significant difference $(p<0.01)$. In post-hoc tests, disc level gap healing was also shown to be different from that of the upper and lower body levels.

We examined the changes in the segmental angle in relation to the opening site. When the opening was in the upper body, a correction angle of $24.1^{\circ}$ was obtained after surgery and that of $23.1^{\circ}$ was observed at the final followup. For lower body gap openings, we obtained $23.6^{\circ}$ of correction after surgery and $21.5^{\circ}$ of correction at the final follow-up. For openings at disc level, however, a $16.8^{\circ}$ angle of correction was obtained after surgery, with $16.2^{\circ}$ of correction at the final follow-up, thus representing the least amount of correction in comparison to those of the other two sites of opening (Table 3). The difference in the average angles of correction for the three groups was statistically significantly $(p=0.03)$. Duncan's post-hoc test showed that the average correction angle of the segmental

Table 2 Preoperative and postoperative radiographic data

\begin{tabular}{|c|c|c|c|c|c|c|}
\hline & Preop & IMPO & IM corr & Final follow-up & Final corr & LOC \\
\hline DSA & $-0.1^{\circ}\left(-7^{\circ}\right.$ to $\left.8^{\circ}\right)$ & $20.1^{\circ}\left(10^{\circ}\right.$ to $\left.31^{\circ}\right)$ & $20.2^{\circ}$ & $18.7^{\circ}\left(11^{\circ}\right.$ to $\left.30^{\circ}\right)$ & $18.8^{\circ}$ & $1.4^{\circ}$ \\
\hline SA & $-12.8^{\circ}\left(-34^{\circ}\right.$ to $\left.1^{\circ}\right)$ & $8.8^{\circ}\left(-10^{\circ}\right.$ to $\left.22^{\circ}\right)$ & $21.6^{\circ}$ & $7.6^{\circ}\left(-12^{\circ}\right.$ to $\left.21^{\circ}\right)$ & $20.4^{\circ}$ & $1.2^{\circ}$ \\
\hline C7 PL (mm) & 149.7 & 42.4 & 107.3 & 45.2 & 104.5 & 2.8 \\
\hline
\end{tabular}

Values in parentheses are ranges

DSA sagittal angle of disc; $S A$ segmental angle; $C 7$ PL C7 plumb line; Preop preoperative; IMPO immediate postoperative; IM corr immediate correction; Final corr final correction; LOC loss of correction 
Table 3 Radiographic data according to anterior opening level

\begin{tabular}{llll}
\hline & $\begin{array}{l}\text { Number of } \\
\text { segment }\end{array}$ & $\begin{array}{l}\text { Final correction } \\
(\mathrm{SA})\end{array}$ & $\begin{array}{l}\text { Gap healing } \\
(\%)\end{array}$ \\
\hline Upper body & 11 & $23.1^{\circ}$ & 100 \\
Lower body & 7 & $21.5^{\circ}$ & 85.7 \\
Disc & 8 & $16.2^{\circ}$ & 12.5 \\
\hline
\end{tabular}

$S A$ segmental angle

angles of the upper body and the lower body levels was different from that at disc level.

VAS scores were improved from 4.5 prior to surgery to 1.4 after surgery and the modified SRS instrument scale showed a substantial increase from an average of 2.9 prior to surgery to an average of 4.2 after surgery.

We did not detect any intraoperative complications. After surgery, however, three patients experienced temporary paralytic ileus recovering spontaneously 3-5 days after conservative treatment. Complications associated with neurological deficits or vascular injuries were not observed in any patients.

\section{Discussion}

Surgical treatment options for advanced ankylosing spondylitis (AS) patients with kyphotic deformity include SPO [7], PSO [8], polysegmental osteotomies [9, 10] or any combination of these $[11,12]$.

Of them, SPO was first reported by Smith-Petersen et al. in 1945 [7] and was initially performed as a single level osteotomy, mainly for the treatment of AS [13-18]. SPO was performed in the lumbar vertebral area at a $30^{\circ}-40^{\circ}$ angle of correction on a single level. However, due to high mortality rates and a high possibility of neurological complications, the method has not been commonly applied $[1,19,20]$ and several modifications of this anterior opening wedge osteotomy have been described [3, 9, 10, $13,21,22]$.

The indication of the SPO technique has gradually expanded and has been applied in various spinal diseases [1]. Polysegmental SPO has been particularly common and it has been possible to achieve a certain level of correction for coronal imbalance through the performance of asymmetric osteotomy $[9,10,21]$. SPO of a single segment has been reported to generally result in $\sim 10^{\circ}$ of correction. In AS patients, SPO could result in a correction of $\sim 40^{\circ}-50^{\circ}$, due to the brittle nature of their bones [1]. However, the significant correction of a single segment could lead to excessive elongation of the anterior column and result in fatal complications such as aortic rupture, paraplegia, and superior mesenteric artery syndrome [19]. Some investigators have observed a loss of correction and pseudarthrosis in large anterior opening gaps [23]. For these reasons, polysegmental osteotomy has been preferred to hyper-extension osteotomy at the single level. Anterior fusion was first mentioned by La Chapelle [13]; according to his study published in 1946, he performed the correction using a two-stage operation. The first surgery involved posterior osteotomy and the second surgery of the anterior column support and the strut bone graft were performed two weeks later. This two-stage system avoids fatal complications such as the rupture of the aorta and allows for safer correction of the deformity with stable bony fusion. Bradford et al. [19] reported that serious complications such as the rupture of the aorta could nonetheless occur after SPO. However, the actual incidence of these complications was not high, and the risk of complications seems to have been overestimated. In our experience, the development of severe complications after SPO has also been relatively rare. McMaster reported a study of SPO performed on 14 AS patients in 1985 in which the correction loss and nonunion were not reported, and an average of $33^{\circ}$ of correction was obtained [24]. Chang et al. [25] reported that successful surgical outcomes could be obtained by posterior correction and fixation without anterior fusion in AS patients with pseudarthrosis. They advocated that the superior fusion abilities of AS patients were the reason that nonunion did not occur. However, nonunion indeed developed in $4.5 \%$ (3/66) of these patients, and revision was required. On the other hand, Bridwell et al. [4] reported that anterior grafting of the disc space may be necessary after achieving substantial correction with an SPO but did not provide specific figures or data regarding this possibility. In addition, he mentioned that producing an anterior gap may make arthrodesis less reliable [5].

All 19 patients in our study underwent a posterior approach alone with SPO and complete fusion was achieved in all patients at a mean period of 5.6 months (3-6.7 months). These results are similar to that (4.2 months) of our previous report [6] in which we performed SPO and anterior interbody fusion for AS patients with pseudarthrosis.

In addition, gap healing appeared to differ according to the anterior opening sites, and we therefore examined the associations among the osteotomy site, the anterior opening area, and the distance from the osteotomy site to the apex of kyphosis. In cases in which the anterior opening developed at the disc level, the distance between the apex of deformity and the osteotomy site was an average of 0.5 levels; however, when the anterior opening was in the upper body, the average was 1.73 levels, and for anterior openings at the lower body, the average was 1.57 levels. When SPO was performed closer to the apex of deformity, the opening in the disc level increased, and when the 
osteotomy site was more distant from the apex of deformity, there was an increase in the development of the anterior opening in the upper and lower body. For osteotomies performed at the apex of deformity, we observed an opening in the disc level in five of the six cases, with only one case having an opening in the lower body. SPO performed at the site close to the apex of deformity may therefore be disadvantageous for gap healing. We considered whether these results could be due to osteoclasis of the upper and lower vertebral body endplates. Osteoblast reactions could be occurring not only in the cortical bone but also in the cancellous bone fracture, and anterior gap healing (ossification) could take place instead of marginal syndesmosis of the disc space. However, the distance from the apex did not show significant differences in relation to either correction angle $(p=0.69)$ or healing $(p=0.09)$.

In our experience, AS patients show characteristic ossifications of the ligament and the joint in the vicinity of the vertebrae, suggesting that excellent bony fusion can be obtained using posterior fusion alone without any anterior column support. Surgery using the anterior approach is stressful to most spinal surgeons, and its necessity must be cautiously discussed. This is especially pertinent given the recent improvements in surgical techniques, the advent of posterior fixation using pedicle screws, strong fixation, and the much improved success rates of fusion in the absence of anterior fusion. The advantages of performing posterior fusion alone without anterior fusion include reductions in the duration of operation, reduction of blood loss and the avoidance of an anterior approach that is relatively unfamiliar to spinal surgeons. This avoidance could potentially decrease morbidity and mortality and may reduce the cost as well as the burden of antero-posterior surgery on patients.

We have also performed combined posterior osteotomy with anterior fusion, although an anterior approach with anterior fusion should perhaps be limited to cases in which correction could not be achieved after posterior osteotomy. The cases that require anterior reconstruction include when bony fusion is delayed for a substantial period after posterior osteotomy or those cases in which pseudarthrosis develops due to nonunion.

We note that our study is limited by the relatively small number of subjects and its retrospective nature involving patients who received corrective osteotomy by posterior approach alone. Additional prospective and comparative studies should be conducted in the future.

\section{Conclusions}

In the correction of kyphotic deformity of AS patients using SPO, an anterior opening angle of more than $10^{\circ}$ is a commonly encountered problem. In our study of subjects presenting with anterior opening angles from $10^{\circ}$ to $32^{\circ}$, we obtained successful fusion and good clinical outcomes without the need for additional anterior interbody fusion. The superior fusion ability associated with AS allowed for successful fusion regardless of complete gap healing of the anterior opening gap.

When osteotomy was performed at the apex of the kyphotic segment, the anterior opening gap developed primarily in the disc space. Improved gap healing and increased correction angles were obtained when the opening was present in the upper or lower body endplates compared to those at the disc space level.

\section{Conflict of interest None.}

Open Access This article is distributed under the terms of the Creative Commons Attribution Noncommercial License which permits any noncommercial use, distribution, and reproduction in any medium, provided the original author(s) and source are credited.

\section{References}

1. Burton DC (2006) Smith-Petersen osteotomy of the spine. Instr Course Lect 55:577-582

2. Bridwell KH (2006) Causes of sagittal spinal imbalance and assessment of the extent of needed correction. Instr Course Lect 55:567-575

3. Voos K, Boachie-Adjei O, Rawlins BA (2001) Multiple vertebral osteotomies in the treatment of rigid adult spine deformities. Spine (Phila Pa 1976) 26:526-533

4. Bridwell KH (2006) Decision making regarding Smith-Petersen versus. pedicle subtraction osteotomy versus vertebral column resection for spinal deformity. Spine (Phila Pa 1976) 31:S171S178

5. Bridwell KH, Lenke LG, Lewis SJ (2001) Treatment of spinal stenosis and fixed sagittal imbalance. Clin Orthop Relat Res $35-44$

6. Kim KT, Lee SH, Suk KS, Lee JH, Im YJ (2007) Spinal pseudarthrosis in advanced ankylosing spondylitis with sagittal plane deformity: clinical characteristics and outcome analysis. Spine (Phila Pa 1976) 32:1641-1647. doi:10.1097/BRS.0b013 e318074c3ce

7. Smith-Petersen MN, Larson CB, Aufranc OE (1969) Osteotomy of the spine for correction of flexion deformity in rheumatoid arthritis. Clin Orthop Relat Res 66:6-9

8. Thomasen E (1985) Vertebral osteotomy for correction of kyphosis in ankylosing spondylitis. Clin Orthop Relat Res 142-152

9. van Royen BJ, de Kleuver M, Slot GH (1998) Polysegmental lumbar posterior wedge osteotomies for correction of kyphosis in ankylosing spondylitis. Eur Spine J 7:104-110

10. Hehne HJ, Zielke K, Bohm H (1990) Polysegmental lumbar osteotomies and transpedicled fixation for correction of long-curved kyphotic deformities in ankylosing spondylitis. Report on 177 cases. Clin Orthop Relat Res 49-55

11. Lagrone MO, Bradford DS, Moe JH, Lonstein JE, Winter RB, Ogilvie JW (1988) Treatment of symptomatic flatback after spinal fusion. J Bone Joint Surg Am 70:569-580

12. Van Royen BJ, De Gast A (1999) Lumbar osteotomy for correction of thoracolumbar kyphotic deformity in ankylosing 
spondylitis. A structured review of three methods of treatment. Ann Rheum Dis 58:399-406

13. La Chapelle EH (1946) Osteotomy of the lumbar spine for correction of kyphosis in a case of ankylosing spondylitis. J Bone Joint Surg Am 28:851-858

14. Briggs H, Keats S, Schlesinger PT (1947) Wedge osteotomy of the spine with bilateral intervertebral foraminotomy; correction of flexion deformity in five cases of ankylosing arthritis of the spine. J Bone Joint Surg Am 29:1075-1082

15. Herbert JJ (1959) Vertebral osteotomy for kyphosis, especially in Marie-Strumpell arthritis; a report on fifty cases. J Bone Joint Surg Am 41-A:291-302 passim

16. McMaster PE (1965) Osteotomy of the spine for fixed flexion deformity. Pac Med Surg 73:314-316

17. Goel MK (1968) Vertebral osteotomy for correction of fixed flexion deformity of the spine. J Bone Joint Surg Am 50:287-294

18. Law WA (1969) Osteotomy of the spine. Clin Orthop Relat Res 66:70-76

19. Bradford DS, Schumacher WL, Lonstein JE, Winter RB (1987) Ankylosing spondylitis: experience in surgical management of 21 patients. Spine (Phila Pa 1976) 12:238-243
20. Kim KT, Suk KS, Cho YJ, Hong GP, Park BJ (2002) Clinical outcome results of pedicle subtraction osteotomy in ankylosing spondylitis with kyphotic deformity. Spine (Phila Pa 1976) 27:612-618

21. Geck MJ, Macagno A, Ponte A, Shufflebarger HL (2007) The Ponte procedure: posterior only treatment of Scheuermann's kyphosis using segmental posterior shortening and pedicle screw instrumentation. J Spinal Disord Tech 20:586-593. doi:10.1097/ BSD.0b013e31803d3b16

22. Simmons EH (1977) Kyphotic deformity of the spine in ankylosing spondylitis. Clin Orthop Relat Res 65-77

23. Weale AE, Marsh CH, Yeoman PM (1995) Secure fixation of lumbar osteotomy. Surgical experience with 50 patients. Clin Orthop Relat Res 216-222

24. McMaster MJ (1985) A technique for lumbar spinal osteotomy in ankylosing spondylitis. J Bone Joint Surg Br 67:204-210

25. Chang KW, Tu MY, Huang HH, Chen HC, Chen YY, Lin CC (2006) Posterior correction and fixation without anterior fusion for pseudoarthrosis with kyphotic deformity in ankylosing spondylitis. Spine (Phila Pa 1976) 31:E408-E413. doi:10.1097/ 01.brs.0000219870.31561.c2 